Widefield ScIENCE AND TeChNOLOGY For the SKA

SKADS CONFERENCE 2009

S.A. Torchinsky, A. van Ardenne, T. van den Brink-Havinga, A.J.J. van Es, A.J. Faulkner (eds.)

4-6 November 2009, Château de Limelette, Belgium

\title{
RFI mitigation at Nançay Observatory: Impulsive Signal Processing
}

\author{
D. Ait-Allal ${ }^{1}$, C. Dumez-Viou ${ }^{1}$, R. Weber $^{2}$, G. Desvignes ${ }^{3}$, I. Cognard ${ }^{3}$, and G. Theureau ${ }^{1,3}$ \\ ${ }^{1}$ Observatoire de Paris, Station de radioastronomie, F-18330 Nanay, France \\ 2 University of Orlans, Site Galile, 12 rue de Blois, 45067 Orlans cedex 2, France \\ ${ }^{3}$ Laboratoire de Physique et Chimie de l'Environnement et de l'Espace, UMR 6115 CNRS, F-45071 Orlans Cedex 02, France
}

\begin{abstract}
Radio astronomy has protected frequency bands for free observations. However, it is often necessary to observe outside of those sanctuaries. For example, it is the case for HI radio-sources with high red-shifts that are observed into radarallocated frequency bands. A radar pulse blanker based on statistical analysis has been implemented in a FPGA. Several tricks has made the implementation possible at a low hardware cost. Pulsar is another kind of impulsive signal which needs specific processing. In the proposed approach, the cyclostationarity is used to discriminate between radio-frequency interference (RFI) pulses and pulsar pulses.
\end{abstract}

\section{Introduction}

Nançay Decimeter Radio telescope, a single antenna telescope, suffers from local ground based Radar RFI while observing red-shifted $\mathrm{H} 1$ recombination lines and pulsars. Pulsar also produces periodic pulses which can be measured with dedicated backends. Radar interferences enter the receiver through low gain side lobes located in the back of the antenna or in the high gain main lobe after direct reflection on an airplane.

Some work has been done to detect and remove such RFI (Ellingson 2003, Dong 2004). However, the complexity of implementation of such algorithms in FPGA logic was a deadlock. To allow HI surveys in this frequency band, an ana$\log$ waveform blanker has been built and operated in Nançay Observatory (Weber et al. 2005). However, only strong pulses could be removed, resulting in unworkable results.

In the first half of this paper, we present a digital radar pulse detector based on a power criterion that triggers waveform blanking. Such detector is the most robust that can be thought from a signal processing point of view, i.e. you bring the least information possible about the RFI to detect. Here, the RFI is detected by an excess of energy during some period of time. Nevertheless, detection performances are usually very poor. Thus, we included several elaborations that do not strongly bear upon implementation cost but provide significant improvements.

In the second half of this paper, we present another RFI detector dedicated to pulsar observations. It will be implemented in the new coherent dedispersion receiver located in the Nançay Radio Telescope also described in this paper.

\section{Pulsed-RFI detection with a power detector}

In order to be efficient, the power detector needs a robust estimation of the decision threshold. Outlayer samples that go past this level are considered as interferences and trigger the blanking of the corresponding data bloc before it enters the spectral estimation process. The issue is that the same bloc of data is used for threshold estimation and detection.

\subsection{Threshold calculation}

Uncorrupted complex data samples follow a normal distribution law since they result from the observation of a radioastronomical source more or less buried under system noise, both ideally following a Gaussian distribution. Thus, the instantaneous power of data samples follows a $\chi^{2}$ distribution with two degree of freedom.

Usually, power detectors are based on a Gaussian model. Actually, there are several disadvantages to use such approach. First, 2 parameters are needed to model the Gaussian distribution, mean and standard deviation. Further more, standard deviation estimation requires prior mean estimation. The error made on the mean estimate will affect the standard deviation estimate as well. Finally, in terms of logical resources and data bus width, the implementation cost of such approach could be an issue (Hampson et al. 2002).

Our approach is based on the $\chi^{2}$ distribution model. So, only one parameter (the mean $\mu$ ) is needed to fully define the signal statistics. Consequently, the threshold value $S$ is calculated like $S=C \mu$ with $C$ a parameter defined by the user. The mean value is recursively estimated (see Fig. 2-1). To make this estimation robust against outliers due to RFI, the strongest samples are systematically discarded. The effect of this clipping on mean estimation can be theoretically derived (Dumez-Viou 2007) and the proper correction is included in the parameter $C$. From this basic scheme, two improvements are proposed to enhance the performances for strong and weak radar pulses.

\subsection{False alarm rate reduction}

In detection systems, a given threshold defines a false alarm rate: samples that should not be classified as RFI, trigger the detector because they lie in the right tail of the uncorrupted 
signal distribution, over the threshold value. This value has to be chosen such that false alarm rate is kept at a reasonably low level. Detecting weaker radar pulses can be achieved with a reduced threshold value leading automatically to an unbearable rate of false alarm. Moreover, for synchronisation reasons (see section 2.3), our detector blanks the whole bloc of data even if only one outlier has been detected in that bloc. This approach worsens the false alarm issue. For example, if the threshold is set to $4 \mu$ to produce a $2.75 \%$ false alarm rate, a 2048 -sample bloc configuration will systematically triggers the detector and the receiver returns no usable results.

To overcome this issue, notice that the time distribution of such false alarms over the whole observation is uniform. Let $\alpha$ be the false alarm rate. Then, the probability to get $N$ consecutive uncorrupted samples that trigger the detector is $\alpha^{N}$. Thus, triggering the blanker if three consecutive samples go past the threshold sets a new false alarm rate of $(2.75 \%)^{3}=2.1 \times 10^{-3} \%$ leading to the blanking of only $6.4 \%$ of the data blocs.

The hardware required to handle the previous modifications is limited to a one-bit wide $(N-1)$-bits deep shift register to store the results of the comparisons between samples and threshold, and a $N$-bits AND gate. This approach $(S=4 \mu$, $N=3, \alpha=2.75 \%$ ) is used to detect strong radar pulses (see Fig. 2-2).

For weak radar pulses, looking at $N$ consecutive samples lead to poor detection performances. Indeed, radar bursts get more and more buried under the system noise. However, tests have shown that counting the number of detection in a time window is better. Thus, for our weak pulse detector (see Fig. 23 ), we set the threshold to $0.8125 \mu$, the time window to 30 samples and the triggering number of detection to 25 . Using binomial law and tabulated $\chi^{2}$ distribution, a false alarm rate of $1.2 \times 10^{-3} \%$ is achieved, resulting in the blanking of $4 \%$ of the data blocs.

In term of implementation, rather than adding the 30 bits for each new sample, we recursively calculate the sum. The balance $(-1,0$ or +1$)$ of ' 1 ' entering and ' 1 ' leaving the shift register is added to the previous count. The hardware now extends to a one-bit wide 30-bits deep shift register, a 1-bit subtractor and a 2-bit/5-bit adder.

Improving a basic power detector implies including some a priori knowledge. We focused on the pulse length that is one of the basic characteristics shared by many radar systems. This way, the detector is still generic but provides better performances for a slight increase in hardware costs.

The performances of our detectors are shown in Fig. 1. The blue curves annotated SP (respectively, WP) corresponds to the behaviour of the strong pulse detector (respectively, weak pulse detector). The results ot two other previous studies have been also included in the figure. Niamsuwan et al. (2005) are based on a simple pulse detector for 2 different setting (PP30 and PP90). Dong et al. (2004) results are based on a much more advanced algorithm that gives better performances for its finest setting (AD05 and AD10). However, it includes a lot of information about a specific radar pulse shape. Any radar pulse whose shape gets to far from the model will not be detected as easily.

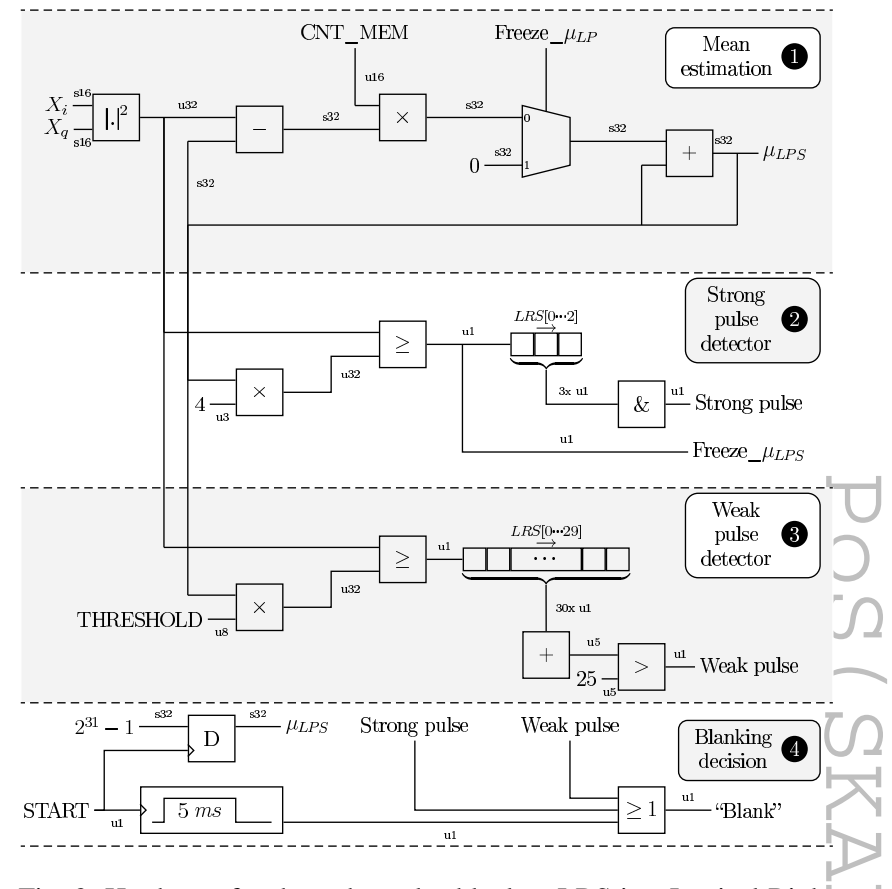

Fig. 2: Hardware for the radar pulse blanker. LRS is a Logical Right Shift unit. $s$. and $u$.. specify the databuses width for signed and unsigned operands.

\subsection{Constrained Blanking}

Blanking data blocs of waveform is not harmless. It was carefully studied in Niamsuwan et al. (2005). In our design, we simply blank data blocs that are synchronized with the data blocs used to perform spectral analysis. Thus, no discontinuities are present in the data bloc sent to the FFT. The drawback of such method is that frequency resolution (i.e. FFT size) imposes the granularity of the blanking. For our design, this leads to an unnecessary amount of blanked data (coarse-grained). For other designs, this might lead to fine-grained blanking that can be adjusted to will with simple glue logic.

\subsection{Hardware ressources}

The hardware that computes the mean estimation and the detection of strong and weak pulses if presented in the figure 2 . The design operates at a maximum sampling rate of $145 \mathrm{Ms} / \mathrm{s}$. However, we only used it at $14 \mathrm{Ms} / \mathrm{s}$ which implies no more than 3 radars in the observing frequency band. The overall blanking rate is about $4 \%$ but this figure could significantly increase for a wider band carrying many more radars. The logical gates used to implement the algorithm occupies $4 \%$ of a 3 Mgates FPGA (Virtex II from Xilinx) and 2 of the 96 $18 \times 18$-multipliers available. The design has been used to observe radio-galaxies with flux densities as low as $5 \mathrm{mJy}$. No radar residuals could be seen on the base line as shown on figure 3.

The next section will present another RFI mitigation application. 


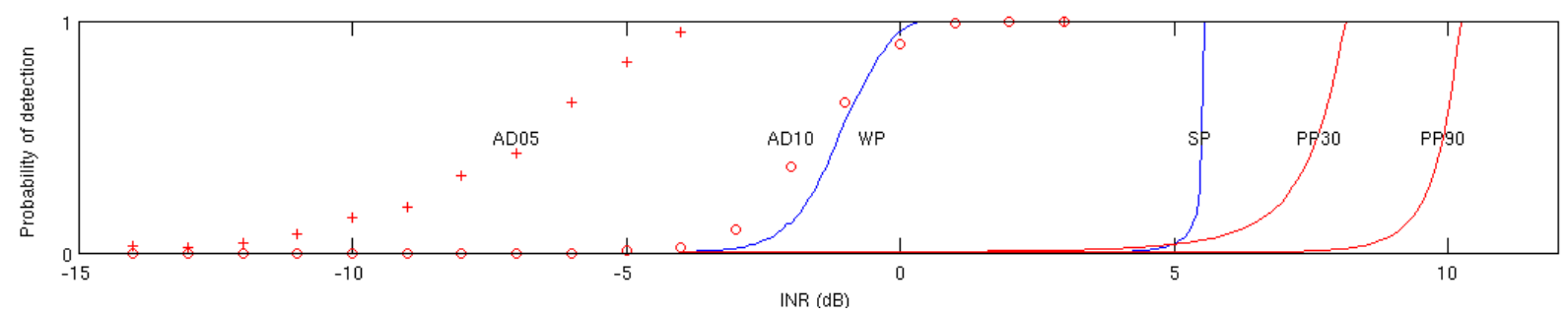

Fig. 1: Probability of detection of several detectors as a function of radar pulse Interference to Noise Ration (INR). AD05 and AD10 refer to Dong et al. (2004). PP30 and PP90 refer to Niamsuwan et al. (2005). WP and SP refer to the proposed hardware.

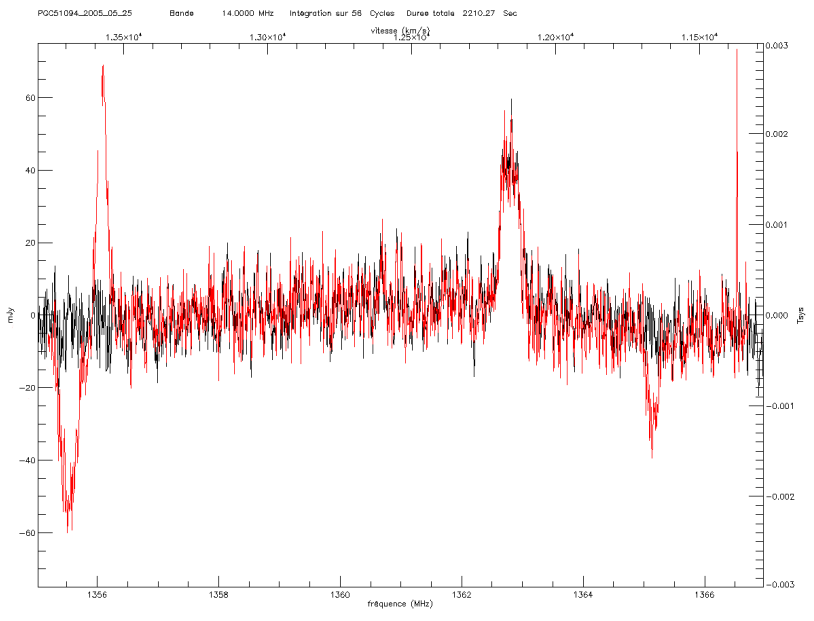

Fig. 3: Observation of PGC051094 with (black) and without (red) blanking.

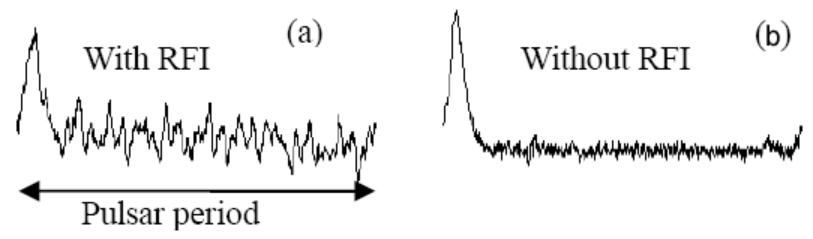

Fig. 4: Pulsar pulse profile: (a) Time representation of the average power pulse profile of the pulsar J0034-0721 after coherent dedispersion but with RFI signals. (b) Time representation of the average power pulse profile of the pulsar J0034-0721 after coherent dedispersion and with RFI signals blanked (with other RFI mitigation scheme).

\section{Pulsar application}

The quality of pulsar observations is limited by RFI generated by various (and growing) Telecommunications activities. Figure 4 gives an example of the distortion induced by RFI on pulsar observation. This section will present the innovative pulsar instrumentation based on Graphical Processing Units (GPU) which has been designed at the Nançay Radio Astronomical Observatory. In addition, first simulated results on RFI cyclostationary detectors, which will be implemented on the system, will be described.

\subsection{Hardware Implementation}

During their travel through the interstellar medium (ISM), these pulsar pulses are progressively attenuated and spread over time (i.e. pulse higher frequency component will arrive earlier than the pulse lower one). This phenomenon, named as dispersion, makes these pulses barely detectable without a dedisperion processing. Figure 5 shows the signal dataflow from the radio telescope to the final de-dispersed pulses. The different steps are described below:

1. The Analog System [Demorest et al. (2007)]: two orthogonal polarization signals from the radio telescope are downconverted through a custom circuit board quadrature downconverter (QDC). Each QDC handles one polarization and performs a quadrature demodulation providing 128 $\mathrm{MHz}$ complex bandwidth which is sent to the SERENDIP5 Spectrometer for digitalization and channelizing.

2. The SERENDIP5 Spectrometer [Demorest et al. (2007)] SERENDIP5 has been designed by the CASPER group in Berkeley. For this application, it contains four 8-bit analog to digital converters (ADCs) that can be clocked up to 200MHz. A logic programmable device (Virtex 2 $\mathrm{XC} 2 \mathrm{~V} 4000$ from Xilinx) is used to perform a 32 channel polyphase filter bank (PFB). The channel output bandwidth is $4 \mathrm{MHz}$ complex. An additional Xilinx device (Virtex 2 $\mathrm{XC2V1000)} \mathrm{is} \mathrm{used} \mathrm{as} \mathrm{a} \mathrm{reconfigurable} \mathrm{backend} \mathrm{processor}$ which can pass data to an independent computer.

3. Data servers: This is the innovative part of the design. The two data severs have two main processings steps. The first one is to read the raw data in from the SERENDIP5 spectrometer and reorganize them from a channel-order to single-channel time ordered chunks. This step is controlled by four micro processors, that send these chunks to four GPUs (Graphic Processing Unit- NVIDIA 88 GTX 128 parallel processors) for the final processings step. The data are then read by the dedispersion program that performs the following tasks:

- Convert raw data from binary to floating point.

- Apply a Fourier transform of appropriate length (8192 bins).

- Multiply by the frequency response of de-dispersion filter and the taper function. The taper function is used to avoid aliasing in the low-pass filtering. The combination of the inverse of the dispersion transfer function and the taper function is known as the chirp function. 


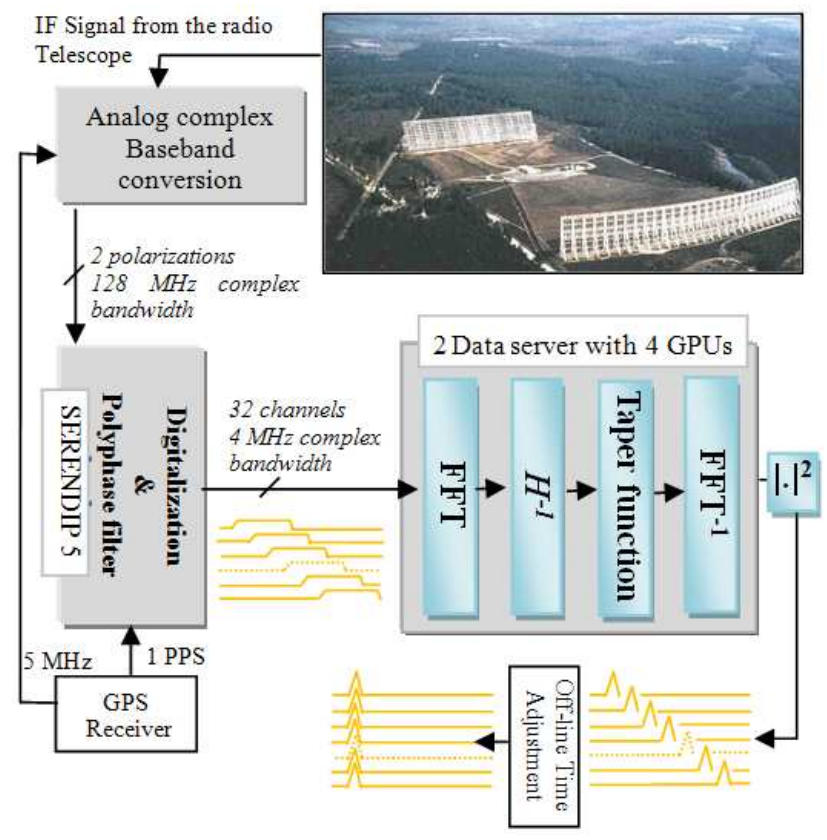

Fig. 5: Description of the instrumentation for pulsar observations which is in operation since July 2008 at Nançay Observatory. The top right hand corner picture is the giant decimetre radio telescope of Nançay. The GPS receiver provides SERENDIP5 clock synchronization through a one pulse per second (1PPS) signal and oscillator synchronization through a $5 \mathrm{MHz}$ reference clock.

- Inverse Fourier transform back to the time domain.

- Detect the data to get power versus time, and crossmultiply polarization terms.

4. Time adjustment: offline resynchronisation of all the channels.

This coherent de-dispersion pulsar receiver is operational since July 2008 at Nançay Observatory and it outperforms the performances of the previous system based on a cluster of $77 \mathrm{bi}-$ processor Athlon $1.2 \mathrm{GHz}$. In the next section, a RFI mitigation technique, which is able to separate pulsar pulses from RFI pulses, is presented.

\subsection{RFI Cyclostationary Detector}

The proposed detector is based on RFI specific properties named cyclostationarity (Serpedin et al. 2005; Gardner et al. 2006; Feliachi et al. 2010). Indeed, most of telecommunications signals present a hidden periodicity which is usually scrambled by the intrinsic signal randomness. For example, this hidden periodic characteristic can be generated by the carrier frequency or the baud rate of the incoming RFI.

In Weber et al. (2007), we have studied the statistics of a cyclostationary detector based on the following criteria:

$$
C_{s}^{\alpha}=\left|\frac{1}{N} \sum_{n=0}^{N-1} s(n)^{2} e^{-i 2 \pi \alpha n}\right|
$$
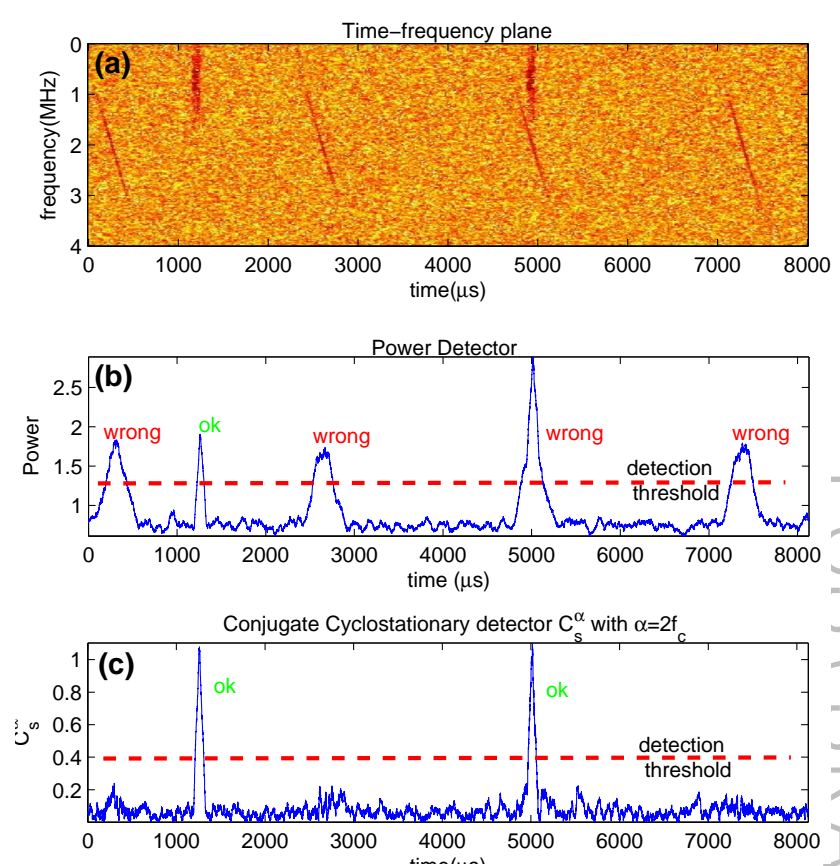

Fig. 6: Detection of impulsive broadband RFI signals (located at $t \simeq 1200 \mu \mathrm{s}$ and $t \simeq 5000 \mu \mathrm{s}$ ) by using the conjugate cyclostationary detector. 4 pulsar pulses are also visible. a) Time-frequency representation. b) Power detector ( $\mathrm{N}=256$ samples). c) Conjugate cyclostationary detector ( $\mathrm{N}=256$ samples).

where $\alpha$ is the frequency related to the hidden periodicity ( $\alpha$ is also called the cyclic frequency), $s(n)$ is the input signal. $C_{s}^{\alpha}$ is called the conjugate cyclostationary detector.

In Figure 6, an application of such detector in the case of pulsar observation is presented. For the simulation, we have considered a BPSK (Binary Phase Shift keying) RFI. The pulsar signal has been generated by a model that we have proposed in Ait Allal et al. (2009). The results show that the cyclostationary detector detects only the bursts while the power detector cannot make the difference between bursts and pulsar.

Depending on where this detector will be implemented in the system, different type of RFI can be detected. Three possibilities have been identified on the system architecture defined on Figure 5:

- At the input of the polyphase filter bank, just after the digitalisation. This configuration is appropriate for impulsive or burst broadband RFI.

- Just after the polyphase filter bank.

- Just after the FFT, in the coherent de-dispersion process. This configuration is appropriate for narrow band and continuous RFI.

By simulation, we have shown that cyclostationary detectors can be a very interesting alternative to power detectors for pulsar observations. 


\section{Conclusions}

Two RFI detectors have been presented, each of them is adapted to a specific RFI context. It is possible to reduce the effect of RFI on radioastronomical observations if a priori knowledge on the RFI can be exploited efficiently.

Acknowledgements. The authors would like to thank Pierre Colom, DS4-T3 task leader, Observatoire de Paris, France, Philippe Ravier, Rachid Harba and Rym Feliachi, all from the University of Orléans, France, for supplying them with helpful materials and remarks. The authors also want to thank the European Commission Framework Program 6, Project SKADS (contract no 011938), the Région Centre for funding part of this work.

\section{References}

Ait Allal, D., Weber, R., Cognard, I., Desvignes, G., Theureau, G. 2009, "RFI mitigation in the context of Pulsar coherent de-dispersion at the Nançay radio astronomical Observatory," in Proc. EUSIPCO 2009, Glasgow, Scotland

Dong, W., Jeffs B.D., Fisher J.R., "Kalman Tracking and Bayesian Detection for Radar RFI Blanking" RFI2004, Workshop in Mitigation of Radio Frequency Interference in Radio Astronomy, Penticton, Canada, july 2004

Demorest, P.B. 2007, "Measuring the Gravitational Wave Background using Precision Pulsar Timing," thesis, Chapter 3, University of California, Berkeley.

Dumez-Viou, C., "Radioastronomical sources restoration from hostile radioelectric environment: Implementation of realtime detectors for dynamic spectra analysis", $\mathrm{PhD}$ thesis, http: //tel . archives-ouvertes. fr/tel-00319939/ $\mathrm{fr} /$, September 2007

Ellingson, S.W. and Hampson G.A., "Mitigation of radar interference in L-band radio astronomy. Astrophysical Journal Supplement Series, 147:167-176, 2003

Feliachi, R., Dumez-viou, C., Weber, R., Boonstra, A.J 2010, in Proc. Wide Field Science and Technology for the SKA, Limelette, Belgium, S.A. Torchinsky et al. (eds), ASTRON, ISBN 978-90-805434-5-4

Gardner, W.A., Napolitano, A., Paura, L. 2006, "Cyclostationarity: Half a century of research," Signal Processing, 86

Hampson, G.A., "Implementation of the Asynchronous Pulse Blanker" june 2002. http: //esl . eng . ohio-state.edu/ rstheory/iip/docserv.html

Lorimer, D., Kramer, M., "Handbook of Pulsar Astronomy," Cambridge, United Kingdom: Cambridge University Press, 2005.

Niamsuwan, N., Johnson, J.T., Ellingson, S.W., "Examination of a simple pulse-blanking technique for radio frequency interference mitigation. RADIO SCIENCE, 40(3), june 2005

Serpedin, E., Panduru, F., Sari, I., Giannakis, G.B. 2005, "Bibliography on cyclostationarity," Signal Processing, 85

Weber, R., Bretteil, S., Coffre, A., Colom, P., DumezViou, C., Couratier, P.,Denis, L., Gérard, E., Kenfack, G., Picard, P., Kerdraon, A., Lecacheux , A., Ravier, P., Thomas and P. Zarka, I., "Theoretical and Practical Radio
Frequency Interference Mitigation Developments at Nançay Observatory" URSI 2005

Weber, R., Zarka, P., Ryabov, V.B., Feliachi, R., Grießmeier, J.M., Denis, L., Kozhyn, R.V., Vinogradov, V.V., Ravier, P. 2007, "Data pre-processing for decametre wavelength exoplanet detection: An example of cyclostationary RFI detector,' in Proc. EUSIPCO 2007, Poznan, Poland 